\title{
Formulações monoestágio para o problema de programação da produção de bebidas dois estágios com sincronia
}

\author{
Deisemara Ferreira ${ }^{a *}$, Bernardo Almada-Lobo ${ }^{\mathrm{b}}$, Reinaldo Morabito ${ }^{\mathrm{c}}$ \\ a*deisemara@icte.uftm.edu.br, UFTM, Brasil \\ balmada.lobo@fe.up.pt, FEUP, Porto, Portugal \\ 'morabito@ufscar.br, UFSCar, Brasil
}

\begin{abstract}
Resumo
Neste trabalho, apresentamos formulações monoestágio para o problema integrado de dimensionamento e sequenciamento de lotes de produção de bebidas dois estágios com sincronia. 0 problema envolve múltiplos produtos, múltiplas máquinas e tempos e custos de troca dependentes da sequência de produção. As formulações monoestágio apresentadas não têm perda de generalidade para representar o problema e, em geral, reduzem as dimensões da formulação dois estágios com sincronia apresentadas em Ferreira, Morabito e Rangel (2009) em termos dos números de variáveis e restrições. Experimentos computacionais preliminares realizados com exemplares baseados em dados reais de uma fábrica de bebidas mostram que os modelos monoestágio propostos são competitivos, quando comparados com o modelo anterior dois estágios com sincronia.
\end{abstract}

Palavras-chave

Problemas integrados de dimensionamento e sequenciamento da produção. Programação da produção de bebidas. Programação inteira mista. Programação matemática.

\section{Introdução}

Uma atividade importante para o bom desempenho de um processo produtivo é a programação da produção, que considera vários fatores como, por exemplo, demanda dos produtos, custos e capacidades de produção, preparo das máquinas, tempos de processamento, entre outros. Em alguns processos produtivos, como na produção de bebidas (refrigerantes, águas, sucos, etc.), há ainda a necessidade de programar e sincronizar mais de um estágio da produção, o que torna esta tarefa ainda mais complexa. Dependendo do ambiente produtivo, é desejável ainda que, em cada estágio, o dimensionamento e o sequenciamento dos lotes de produção sejam definidos simultaneamente, uma vez que estas decisões são dependentes uma da outra e ambas podem consumir altos níveis de utilização de capacidade de produção.
A programação da produção na indústria de bebidas contempla todos os fatores descritos anteriormente. Este processo de produção inclui duas grandes etapas: o preparo do xarope (sabor da bebida) e o envase da bebida pronta. Estes dois estágios têm que ser sincronizados para garantir o correto fluxo da bebida. Uma vez que o gargalo da produção pode variar dos tanques da bebida para as linhas de envase, um programa de produção factível deve incorporar ambos os recursos simultaneamente. Note-se que bebidas como refrigerantes são perecíveis e por isto não podem ser produzidos e estocados em grandes quantidades. Por outro lado, os tempos de troca de produtos são dependentes da sequência e podem ser altos, por exemplo, a produção de um refrigerante diet seguida da produção de um refrigerante normal consome 30 minutos de tempo para limpeza nas 
linhas de envase. Se estas bebidas forem produzidas na ordem inversa, refrigerante normal e depois diet, o tempo de limpeza seria de aproximadamente 4 horas. Assim, para um melhor aproveitamento da capacidade de produção das linhas, o dimensionamento e o sequenciamento da produção de bebidas devem ser feitos de forma simultânea.

$\mathrm{Na}$ literatura há diversos trabalhos que modelam matematicamente apenas as decisões de dimensionamento dos lotes (e.g., DREXL; KIMMS, 1997; FRANÇA; ARMENTANO; TOLEDO, 1999; BRAHIMl et al., 2006; TOLEDO; ARMENTANO, 2006), trabalhos que modelam apenas as decisões de sequenciamento da produção (e.g., MANNE, 1960; PINEDO, 1995; CHENG; DING; LIN, 2004), e trabalhos que integram o dimensionamento e o sequenciamento dos lotes em um mesmo modelo matemático (e.g., FLEISCHMANN; MEYR, 1997; DREXL; KIMMS, 1997; CLARK; CLARK, 2000; KARIMI; GHOMI; WILSON, 2003; CLARK; MORABITO; TOSO, 2010). Aplicações destes modelos têm sido estudadas para auxiliar nas decisões de programação da produção de diversas indústrias. Alguns exemplos de estudos em empresas brasileiras e portuguesas aparecem em Araujo, Arenales e Clark (2008), Santos-Meza, Santos e Arenales (2002), Toso, Morabito e Clark (2009), Luche, Morabito e Pureza (2008), AlmadaLobo et al. $(2008,2010)$ aplicados, respectivamente, nos setores de fundição, nutrição animal, grãos eletrofundidos e vasilhame de vidro.

Com relação a estudos no setor de bebidas, exemplos aparecem em Rangel e Ferreira (2003), Clark (2003), Castro e Pizzolato (2005), Toledo et al. (2007, 2008) e Ferreira et al. (2008), Ferreira, Morabito e Rangel (2008, 2009, 2010). Em Ferreira, Morabito e Rangel (2008, 2010), foi estudado um problema de dimensionamento e sequenciamento da produção de refrigerantes com tempos e custos de troca dependentes da sequência em uma fábrica de bebidas de pequeno porte, por meio de um modelo de otimização baseado no modelo monoestágio GLSP (General Lot Sizing and Scheduling Problem) (FLEISCHMANN; MEYR, 1997). 0 modelo considera apenas o estágio de produção de envase da bebida, tratado como gargalo da produção, com uma única linha de envase. Heurísticas do tipo relax and fix foram propostas para resolver o modelo.

Toledo et al. $(2007,2008)$ propõem um modelo de otimização inteira mista para o caso mais geral do problema de bebidas, em que é necessário programar e sincronizar (aspecto importante em fábricas de médio e grande porte) os estágios de preparo do xarope e envase da bebida, considerando vários tanques no preparo do xarope e várias linhas paralelas de envase.
Devido à complexidade e dimensão deste modelo (que envolve cerca de 65 conjuntos de restrições), foram propostas abordagens heurísticas de solução por meio de algoritmos genéticos e meméticos. Em Ferreira, Morabito e Rangel (2009), também foi proposto um modelo de otimização inteira mista para o problema dois estágios com sincronia e múltiplas máquinas, denominado Modelo Dois Estágios Multimáquinas (P2SMM). Nele são admitidas hipóteses simplificadoras em relação ao modelo proposto em Toledo et al. (2007), como a dedicação de tanques a linhas de envase. Ferreira, Morabito e Rangel (2009) explicam que, em visitas a fábricas de bebidas, o número de tanques é em geral maior que o número de linhas de envase, o que permite esta simplificação. É discutido ainda, que esta simplificação permitiu o desenvolvimento de um modelo mais tratável do que o modelo proposto por Toledo et al. (2007). Para resolvê-lo, foi estudada uma abordagem de solução baseada em uma estratégia de relaxação (ER) do modelo, combinada com heurísticas relax and fix, ER_G21. Uma comparação das abordagens em Toledo et al. (2007, 2008) e Ferreira, Morabito e Rangel (2009) pode ser encontrada em Ferreira et al. (2008).

No presente trabalho, apresentamos duas formulações monoestágio para representar o problema de programação de bebidas multiprodutos, multimáquinas e dois estágios com sincronia. Experimentos computacionais realizados com exemplares de tamanho moderado e exemplares de grande porte, baseados em dados reais de uma fábrica de bebidas, mostram que um dos modelos propostos é bem competitivo quando comparado com o modelo P2SMM e a abordagem heurística ER_G21, que é uma estratégia de relaxação combinada com uma heurística do tipo relax and fix denominada G21. Além disso, para um destes exemplares, para o qual dispomos do programa de produção utilizado pela empresa, este modelo obteve uma solução melhor do que a da empresa.

Este artigo está organizado conforme segue. $\mathrm{Na}$ próxima seção, descrevemos resumidamente o processo de produção e a programação da produção de refrigerantes e, por conveniência, também revisamos brevemente o modelo P2SMM. Na Seção 3, apresentamos as formulações monoestágio para o problema, denominadas R1 e R2. Na Seção 4, analisamos os resultados computacionais de experimentos realizados com exemplares baseados em dados reais. E, finalmente, na Seção 5, apresentamos as considerações finais e propostas de trabalhos futuros. 


\section{Programação da produção de bebidas e modelagem do problema}

\subsection{Processo de produção de refrigerantes}

Conforme mencionado, o processo de produção de bebidas possui dois estágios principais, que são o preparo do xarope (sabor) e o envase da bebida pronta. No primeiro estágio, o xarope é preparado em tanques especiais que possuem hélices para agitar o líquido. Uma quantidade mínima de xarope, suficiente para cobrir as hélices, deve ser preparada para garantir sua homogeneidade. Há necessidade de preparar o tanque (limpeza) antes de seu uso. Este preparo é dependente da sequência de produção de xaropes e ocorre mesmo entre trocas de xaropes de mesmo sabor. 0 tempo de troca no tanque é então o tempo de limpeza do tanque, somado ao tempo de preparar o xarope. Após o preparo, o xarope é enviado para as linhas de envase, se estas estiverem prontas. Neste segundo estágio de produção, independente do número de tanques, cada linha de envase recebe xarope de apenas um tanque por vez; porém, um tanque pode enviar xarope para mais de uma linha, simultaneamente, se elas estiverem envasando o mesmo sabor de bebida. 0 tempo de troca na linha é considerado o tempo de limpeza da linha, se o novo item a ser produzido for de sabor diferente, e/ ou o tempo de ajustes mecânicos, se o novo item a ser produzido utilizar um vasilhame de tamanho diferente. Caso contrário, esse tempo de troca e/ou ajuste da linha de envase é nulo.

Outro fator importante na programação da produção de bebidas, além dos tempos e custos de trocas dependentes da sequência, é a sincronia entre os estágios de preparo de xarope e envase da bebida. $\mathrm{Na}$ prática, se o tanque não estiver com o xarope pronto para ser enviado para a linha de envase, esta deve aguardar até que o xarope esteja pronto. Do mesmo modo, o tanque só pode iniciar o envio de xarope para a linha de envase se ela estiver preparada. Assim, podem ocorrer esperas da linha de envase pelo tanque e do tanque pela linha de envase. As Figuras 1 e 2 representam situações em que o tanque e a linha de envase estão dedicados e programados, porém, na Figura 1, não há sincronia de produção entre os estágios. Note nas figuras que os tipos de refrigerantes são representados por números (1-3) e os tipos de xaropes por letras (a e b). Os lotes de produção (retângulos) determinam os tamanhos dos lotes, e o espaço entre eles define os tempos de troca de um xarope para outro no tanque, ou a troca de um refrigerante para outro na linha de envase.
No exemplo da Figura 1, se forem consideradas estas esperas, ou seja, se o tempo de troca for considerado como sendo o maior tempo entre a troca do item na linha e o xarope a ser preparado no tanque, a programação seria como está representado na Figura 2. Os retângulos de cor preta na Figura 2 são a diferença entre o tempo de troca de produtos da linha e o tempo de troca de xaropes no tanque. Note que a inclusão dos tempos de espera equivale a considerar na programação o maior tempo de troca entre produtos na linha e xaropes no tanque. Uma programação da produção não sincronizada, ou seja, sem a consideração das diferenças entre os tempos de troca dos dois estágios, pode levar a uma programação inviável na prática, uma vez que os tempos de espera podem consumir parte significativa da capacidade de produção. Para mais detalhes do processo de produção de refrigerantes, o leitor pode consultar, por exemplo, Toledo et al. (2007, 2008) e Ferreira et al. (2008), Ferreira, Morabito e Rangel (2009).

\subsection{Modelo dois estágios multimáquinas - $P 2 S M M$}

Por conveniência, a seguir, apresentamos resumidamente o modelo P2SMM proposto em Ferreira, Morabito e Rangel (2009). Nele os tanques se encontram dedicados às máquinas, havendo, portanto, igual número de ambos os recursos produtivos. Os parâmetros: número total de refrigerantes (itens), xaropes, linhas de envase (máquinas) e tanques, períodos e subperíodos (i.e. número total de preparos) são designados, respectivamente, pelas letras maiúsculas $J, L, M, T$ e $N$. Sejam os índices definidos como $i, j \in(1, \ldots, J)$ itens; $t \in(1, \ldots, T)$ períodos; $s \in(1, \ldots, M)$ subperíodos; $k, l \in(1, \ldots, L)$ sabor dos



Capacidade disponível

Figura 1. Programação não sincronizada (FERREIRA; MORABITO RANGEL, 2009).

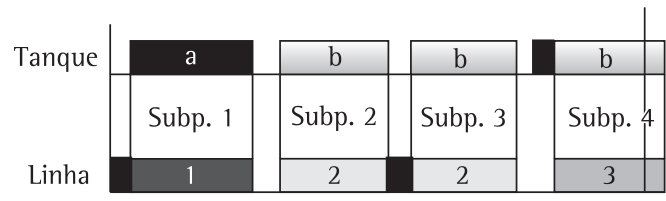

Capacidade disponível

Figura 2. Programação sincronizada (FERREIRA; MORABITO; RANGEL, 2009). 
xaropes; $m \in(1, \ldots, M)$ máquinas e tanques. Suponha que os seguintes conjuntos são conhecidos: $S_{t}$ conjunto dos subperíodos do período $t$ (i.e., $S_{t}$ é um subconjunto de $(1, \ldots, M)$, satisfazendo $\sum_{t=1}^{T}\left|S_{t}\right|=N ; \lambda_{j}$ conjunto de todas as máquinas que podem produzir o item $j ; \alpha_{m}$ conjunto de todos os refrigerantes que podem ser produzidos na máquina $m ; \beta_{m}$ conjunto de todos os xaropes que podem ser preparados no tanque $m$; $\gamma_{m l}$ conjunto de todos os refrigerantes que podem ser produzidos na máquina $m$ e utilizam o xarope $l$. Os dados e as variáveis abaixo com o sobrescrito $l$ se referem ao estágio de xaroparia do processo de produção, e os com o sobrescrito $l l$ se referem ao estágio de envase. Note que os custos considerados, bem como os tempos de troca, são independentes das máquinas ou tanques.

Dados

$d_{j t}=$ demanda do item $j$ no período $t$;

$h_{j}=$ custo de estocar uma unidade do item $j$;

$g_{j}=$ custo de atrasar a entrega de uma unidade do item $j$;

$c_{k l}^{l}=$ custo de fazer a troca do xarope $k$ para $l$;

$c_{i j}^{l l}=$ custo de fazer a troca do item $i$ para $j$;

$b_{k l}^{l}=$ quantidade consumida de tempo para fazer a troca do xarope $k$ para o $l$;

$b_{i j}^{l l}=$ quantidade consumida de tempo para fazer a troca de produção do item $i$ para $j$;

$a_{m j}^{l l}=$ quantidade consumida de tempo para produção de uma unidade do item $j$ na máquina $m$;

$K_{m l}^{l}=$ lote máximo do xarope $I$ no tanque $m$;

$K_{m t}^{l l}=$ capacidade de tempo disponível na máquina $m$ para envase no período $t$;

$q_{m l}^{l}=$ quantidade mínima do xarope $l$ a ser preparada no tanque $m$;

$r_{j l}=$ quantidade consumida de xarope $l$ para produção de uma unidade do item $j$;

$P_{t}=$ primeiro subperíodo do período $t$;

$l_{j 0}^{+}=$estoque inicial do item $j$;

$l_{j 0}^{-}=$atraso inicial do item $j$;

$y_{m / 0}^{l}=$ preparo do xarope $I$ no tanque $m$ no início do horizonte de planejamento;

$y_{m j 0}^{l l}=$ preparo do item $j$ na máquina $m$ no início do horizonte de planejamento;

$\sigma_{j}=$ xarope utilizado para produzir o item $j$.

Variáveis:

$l_{j t}^{+}=$estoque do item $j$ no período $t$;

$I_{j t}^{-}=$quantidade em atraso do item $j$ no período $t$;

$\chi_{m j s}^{\prime \prime}=$ produção da máquina $m$ do item $j$ no subperíodo $s$;
$V_{m s}^{l l}=$ tempo que a máquina $m$ no subperíodo $s$ ficou aguardando o preparo do tanque;

$y_{m l s}^{l}=1$ se há produção no tanque $m$ do xarope I no subperíodo s; 0 caso contrário;

$y_{m j s}^{\prime l}=1$ se a linha $m$ está preparada para produção do item $j$ no subperíodo $s ; 0$ caso contrário;

$z_{m k l s}^{l}=1$ se há troca no tanque $m$ do xarope $k$ para o xarope $I$ no subperíodo $s$; 0 caso contrário;

$z_{m i j s}^{I l}=1$ se há troca na máquina $m$ do item $i$ para o item $j$ no subperíodo $s ; 0$ caso contrário.

0 modelo P2SMM para a programação da produção dois estágios com sincronia é definido:

$\operatorname{Min} \mathrm{Z}=\sum_{j=1}^{\mathrm{J}} \sum_{\mathrm{t}=1}^{T}\left(h_{j} l_{j t}^{+}+g_{j} J_{j t}^{-}\right)+\sum_{m=1}^{M} \sum_{s=1}^{N} \sum_{k \in \beta_{m}} \sum_{l \in \beta_{m}} c_{k l}^{l} z_{m k l s}^{l}+$

$+\sum_{m=1}^{M} \sum_{s=1}^{N} \sum_{i \in \alpha_{m}} \sum_{j \in \alpha_{m}} c_{i j}^{\prime \prime \prime} z_{m i j s}^{\prime \prime}$

Sujeito a:

Estágio 1 (Xaroparia)

$\sum_{j \in \gamma_{m l}} r_{j l} x_{m j s}^{\prime \prime} \leq K_{m l}^{\prime} y_{m l s}^{l}, m=1, \ldots, M, l \in \beta_{m} s=1, \ldots, N ;$; (2)

$\sum_{j \in \gamma_{m l}} r_{j l} x_{m j s}^{\prime \prime} \geq q_{m l s}^{l} y_{m l s}^{\prime}, m=1, \ldots, M, l \in \beta_{m}, s=1, \ldots, N ;$;

$\sum_{l \in \beta_{m}} y_{m l /(s-1)}^{\prime} \geq \sum_{l \in \beta_{m}} y_{m l s}^{\prime} m=1, \ldots, M, t=1, \ldots, T, s \in S_{t} \mid\left\{P_{t}\right\} ;(4)$

$z_{m k l s}^{\prime} \geq y_{m k(s-1)}^{\prime}+y_{m l s}^{\prime}-1 m=1, \ldots, M, k, l \in \beta_{m}, s=1, \ldots, N$; (5)

$z_{m k l s}^{l} \geq \sum_{j \in \gamma_{m k}} y_{m j(s-1)}^{l l}+y_{m l s}^{l}-1$

$m=1, \ldots, M, k, l \in \beta_{m}, t=2, \ldots, T, s=P_{t} ;$

$\sum_{k \in \beta_{m}} \sum_{l \in \beta_{m}} z_{m k l s}^{l} \leq 1 m=1, \ldots, M, s=1, \ldots, N$;

\section{Estágio ll (Envase)}

$l_{j(t-1)}^{+}+l_{j t}^{-}+\sum_{m \in \lambda_{j}} \sum_{s \in S_{t}} x_{m j s}^{\prime \prime}=l_{j t}^{+}+l_{j(t-1)}^{-}+$

$+d_{j t}, j=1, \ldots, J, t=1, \ldots, T$;

$\sum_{j \in \alpha_{m}} \sum_{s \in S_{t}} a_{m j}^{l l} x_{m j s}^{\prime \prime}+\sum_{i \in \alpha_{m}} \sum_{j \in \alpha_{m}} \sum_{s \in S_{t}} b_{i j}^{l l} z_{m i j s}^{\prime \prime}+$

$+\sum_{s \in S_{t}} v_{m s}^{l \prime} \leq K_{m t}^{l \prime} m=1, \ldots, M, t=1, \ldots, T ;$

$v_{m s}^{l l} \geq \sum_{k \in \beta_{m}} \sum_{l \in \beta_{m}} b_{k l}^{l} z_{m k l s}^{l}-\sum_{i \in \alpha_{m}} \sum_{j \in \alpha_{m}} b_{i j}^{l l} z_{m i j s}^{l l}$

$m=1, \ldots, M, \quad s=1, \ldots, N$;

$x_{m j s}^{\prime l} \leq \frac{K_{m t}^{\prime l}}{a_{j}^{l l}} y_{m j s}^{\prime \prime} m=1, \ldots, M, j \in \alpha_{m}, s=1, \ldots, N ;$

$\sum_{j \in \alpha_{m}} y_{m j s}^{\prime \prime}=1, m=1, \ldots, M, \quad s=1, \ldots, N$; 
$z_{m i j s}^{\prime \prime} \geq y_{m i(s-1)}^{\prime l}+y_{m j s}^{\prime \prime}-1, m=$

$=1, \ldots, M, i, j \in \alpha_{m}, s=1, \ldots, N$;

$$
\sum_{i \in \alpha_{m}} \sum_{j \in \alpha_{m}} z_{m i j s}^{l l} \leq 1, m=1, \ldots, M, s=1, \ldots, N \text {; }
$$

$l_{j t}^{+}, l_{j t}^{-} \geq 0, j=1, \ldots, J, t=1, \ldots, T ; x_{m j s}^{\prime l}$,

$v_{m s}^{l l}, z_{m i j s}^{l l}, z_{m k l s}^{l} \geq 0 ; y_{m j s}^{l l}, y_{m l s}^{l}=0 / 1, m=$

$=1, \ldots, M, i$ e $j \in \alpha_{m}, k$ e $l \in \beta_{m}, s=1, \ldots, N$.

A função objetivo (1) minimiza os custos de estoque, atraso e troca. A restrição (2) junto com a restrição (3) garantem os lotes máximos e mínimos, respectivamente, do xarope $l$. A restrição (4) ordena a produção em subperíodos consecutivos dentro de cada período. A restrição (5), equivalente à restrição (13) do estágio 11, controla a troca de xarope no tanque. Porém, esta restrição não controla as trocas entre períodos se o último subperíodo do período anterior for ocioso, sendo então necessária a restrição (6) para contabilizar estas trocas. A restrição (7) é semelhante à (14), e limita o número de trocas por subperíodo. A restrição (8) diz respeito ao balanceamento entre estoque e produção. A restrição (9) garante que a soma do tempo de produção, o tempo gasto para as trocas de refrigerantes, e o tempo de espera da máquina não excederão a capacidade de tempo do período $t$ da máquina $m$. Sendo que o tempo de espera da máquina é a diferença entre os tempos de troca do tanque e os tempos de troca na máquina, restrição (10). A restrição (11) garante que não haverá produção caso a máquina $m$ não esteja preparada. A restrição (12) estabelece que a máquina sempre esteja preparada para produzir exatamente um refrigerante por subperíodo. A restrição (13) controla a troca de refrigerantes. A minimização dos custos de troca na função objetivo define que na solução ótima haja apenas uma troca por subperíodo. Porém, a restrição (14) é mantida, pois facilita a solução do modelo, eliminando soluções em que há mais de uma troca. A restrição (15) define o domínio das variáveis.

\section{Reformulação monoestágio do modelo P2SMM}

Um aspecto relevante deste artigo é mostrar que é possivel desenvolver formulações do tipo monoestágio para o problema, que aproveitem o fato de cada linha possuir um tanque de xarope a ela dedicado. Como dito anteriormente, toda vez que um novo lote de bebidas está designado para ser produzido, deve ocorrer um preparo no tanque e/ou na linha. Se a bebida for a mesma da anteriormente produzida, então há necessidade apenas de preparar o xarope no tanque. Caso contrário, ou seja, se um novo item é produzido, além do preparo do xarope, há o preparo da linha que pode dever-se à sua limpeza e/ou ajustes mecânicos para troca de vasilhame.

Note que o preparo dos tanques sempre ocorre. Mesmo que a bebida a ser produzida seja a mesma, é necessário misturar os ingredientes para produzir o xarope a ser envasado. Isto implica que os elementos da matriz de tempos de troca no tanque são todos positivos. Por esta razão no modelo P2SMM não pode ser definida uma restrição do tipo (12) para o estágio 1 , para que não sejam contabilizadas trocas, mesmo quando não há produção. Ao contrário do que ocorre nos tanques, nas linhas, o tempo/custo de um item para ele próprio é nulo. Daí o modelo P2SMM manter a configuração da linha durante todo o horizonte de planejamento (restrição (12)), recorrendo a uma matriz de tempos/custos de itens que é nula na sua diagonal. Assim, embora a restrição (12) garanta que a linha está preparada em todos os subperíodos, na solução ótima não haverá tempos ou custos contabilizados, pois a linha é mantida preparada com o último item produzido, e as trocas (fictícias, existem apenas por uma questão de modelagem) dele para ele mesmo nos subperíodos ociosos não geram tempos nem custos de troca positivos. Note que no modelo original GLSP de Fleischmann e Meyr (1997) é exigido que $b_{j j}^{\prime \prime}=0$ e $c_{j j}^{\prime \prime}=0$ para que a máquina seja em cada microperíodo configurada para um determinado produto.

Estas particularidades da matriz de tempos de troca de xarope e das restrições do tipo (12) é que impedem que o tempo de troca de uma bebida $i$ para uma bebida $j$ seja tomado como o máximo entre os tempos de troca de $i$ para $j$ na linha e dos respectivos xaropes $\sigma_{i}$ para $\sigma_{j}$ no tanque, pois se estaria penalizando a função objetivo e o consumo de recursos de uma forma inapropriada. No entanto, a substituição dos tempos de troca por outro que resulte do máximo entre os tempos de troca de linhas e tanques é benéfica para uma eventual formulação monoestágio. Neste trabalho, propomos formulações nas quais os tempos de troca são considerados desta forma.

Dado que cada linha possui um tanque dedicado, os lotes mínimos e máximos de xarope, que definem os tamanhos mínimos e máximos de produção, podem ser adaptados para lotes mínimos e máximos de produção nas linhas. Com isto, é possível formular o problema como um modelo monoestágio de produção, facilitando o seu entendimento e, em geral, reduzindo o número total de variáveis e restrições, bem como o tempo computacional necessário para resolvê-lo. 
Ao considerar apenas um parâmetro para caracterizar o tempo de troca de uma bebida $i$ para uma $j$, independentemente de se estar na linha ou no tanque, o tempo de espera acrescentado na restrição de capacidade (9) fica definido no tempo de troca de bebida. Além disto, é necessário definir apenas o sequenciamento das linhas, pois o trade-off entre priorizar o sequenciamento dos xaropes ou bebidas para minimizar custos de troca está embutido em uma única matriz. E assim, fica definida a sincronia entre o estágio 1 e o estágio 11 , pois a linha (tanque) não inicia o envase (ou o envio do xarope) antes que o outro estágio esteja pronto. Desta forma, as restrições do tipo (12) são mantidas, pois elas garantem que as trocas serão contabilizadas nas restrições (13). Os tempos e custos de troca de produção em subperíodos ociosos são anulados com o auxílio de um novo conjunto de variáveis. 0 modelo monoestágio proposto é detalhado a seguir.

\subsection{Formulação monoestágio $R 1$}

0 modelo monoestágio denominado R1 considera a sincronia entre os xaropes do estágio 1 e os lotes de bebida do estágio 11 através de uma única matriz em um único "estágio" de produção, no caso o estágio 11 de envase. Ao contrário do modelo P2SMM, nesta formulação R1 não é preciso diferenciar as variáveis e os parâmetros associados ao tanque (sobrescrito 1) dos associados à linha (sobrescrito 11). Os parâmetros de lotes máximos e mínimos dos xaropes são transformados em lotes de bebida pronta. Desta forma, definimos os parâmetros:

- $U B_{m j}=$ lote de refrigerante $j$, que se pode envasar com um tanque $m$ cheio do xarope, ou seja, $U B_{m j}=K_{m \sigma_{j}}^{l} / r_{j \sigma_{j}} ;$

- $L B_{m j}=$ lote de bebida $j$ que se envasa com o lote mínimo de xarope do tanque $m$ que deve ser preparado, ou seja, $L B_{m j}=q_{m \sigma_{j}} / r_{j \sigma_{j}}$;

- $b_{i j}=$ máximo entre o tempo de troca da bebida $i$ para $j$ na linha e o tempo necessário para a troca do xarope $\sigma_{i}$ para $\sigma_{j}$ no tanque, ou seja, $b_{i j}=\max \left\{b_{i j}^{l l}, b_{\sigma_{i} \sigma_{j}}^{l}: i, j \in J\right\}$;
- $c_{i j}=$ máximo entre 0 custo de troca da bebida $i$ para $j$ na linha e o custo de troca do xarope $\sigma_{i}$ para $\sigma_{j}$ no tanque, ou seja, $c_{i j}=\max \left\{c_{i j}^{l l}, c_{\sigma_{i} \sigma_{j}}^{l}: i, j \in J\right\}$. Convém salientar que, no caso da programação da produção de bebidas, os custos de troca em geral são relativos ao custo de oportunidade quando se deixa de produzir ao parar as linhas ou tanques para o preparo. Assim, o custo $c_{i j}$ é o custo relativo ao tempo total de parada. Nos casos em que os custos de troca em cada estágio representem, por exemplo, custos do processo ou produtos de limpeza, o custo $c_{i j}$ deve ser a soma entre os custos de cada estágio.

Considere que as variáveis de produção, estoque, atraso, e troca de bebidas nas linhas são as mesmas do modelo P2SMM, porém, daqui em diante, ignora-se o sobrescrito 11. Definimos a variável binária $w_{m j s}$, mencionada anteriormente, como:

$W_{m j s}=\left\{\begin{array}{c}1, \text { se a máquina } m \text { se encontra configurada } \\ \text { para o item } j \text { em um subperíodo } s \text { ocioso } \\ 0, \text { caso contrário. }\end{array}\right.$

Esta variável anula as variáveis de preparo e troca em períodos em que não há produção, ou seja, em períodos em que os tempos de troca não devem ser contabilizados. Por exemplo, a Figura 3, a seguir, representa um sequenciamento em 10 subperíodos de uma linha $m$ que envasa 3 itens diferentes. Há produção nos subperíodos de 1 a 4, os subperíodos de 5 a 10 são ociosos e estão preparados com o último item produzido, no caso o item 3.

Nos subperíodos em que há produção, a variável $w_{m j s}$ é nula para todos os itens. Na solução ótima (caso exista), após um subperíodo ocioso, todos os seguintes também o serão e estarão preparados com o último item produzido. Assim, no exemplo, a partir do subperíodo 5 , a variável $w_{m 35}$ passa a assumir valor 1 para $j=3$, que é o último item produzido no período, e é nula para todos os outros itens $(j \neq 3)$ neste subperíodo. 0 mesmo ocorre nos subperíodos consecutivos de 6 a 10 que serão todos ociosos. 0 modelo R1 é definido por:

Linha de envase

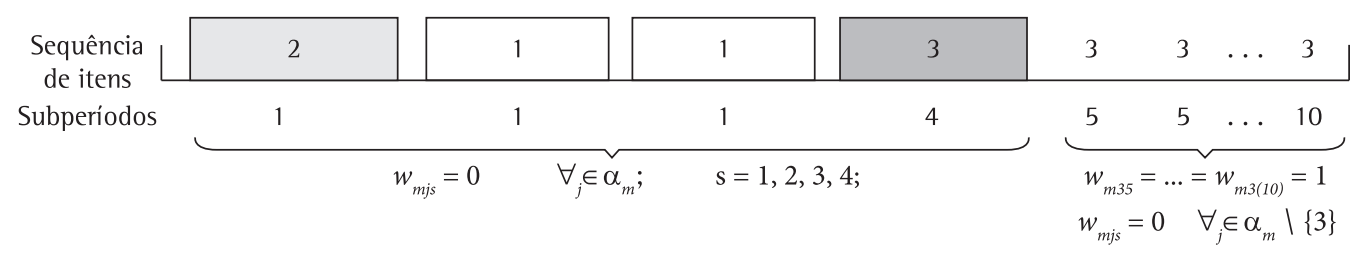

Figura 3. Utilização da variável $w_{m j s}$. 


$$
\begin{aligned}
& \text { Min } \mathrm{Z}=\sum_{j=1}^{J} \sum_{\mathrm{t}=1}^{T}\left(h_{j} l_{j t}^{+}+g_{j} l_{j t}^{-}\right)+ \\
& +\sum_{m=1}^{M} \sum_{s \in S_{t}}\left(\sum_{i \in \alpha_{m}} \sum_{j \in \alpha_{m}, i \neq j} c_{i j} z_{m i j s}+\sum_{j \in \alpha_{m}} c_{i j}\left(z_{m i j s}-w_{m j s}\right)\right)
\end{aligned}
$$

Sujeito a:

$$
\begin{aligned}
& l_{j(t-1)}^{+}+l_{j t}^{-}+\sum_{s \in S_{t}} \sum_{m \in \lambda_{j}} x_{m j s}=l_{j t}^{+}+l_{j(t-1)}^{-}+d_{j t}, j=1, \ldots, J, t=1, \ldots, T ; \\
& x_{m j s} \leq U B_{m j}\left(y_{m j s}-w_{m j s}\right), m=1, \ldots, M, j \in \alpha_{m}, s=1, \ldots, N ; \\
& x_{m j s} \geq L B_{m j}\left(y_{m j s}-w_{m j j}\right), m=1, \ldots, M, j \in \alpha_{m}, s=1, \ldots, N ;(19) \\
& \sum_{s \in S_{t}} \sum_{j \in \alpha_{m}} a_{m j} x_{m j s}+\sum_{s \in S_{t}}\left(\sum_{\substack{i \in \alpha_{m} \\
\sum_{j \in \alpha_{m}}}} b_{i j} Z_{m i j s}+\sum_{j \in \alpha_{m}} b_{j j}\left(z_{m i j s}-w_{m j s}\right)\right) \leq \\
& \leq K_{m t}, m=1, \ldots, M, t=1, \ldots, T ;
\end{aligned}
$$$$
\sum_{j \in \alpha_{m}} y_{m j s}=1, m=1, \ldots, M, s=1, \ldots, N
$$$$
w_{m j(s-1)} \leq w_{m j s} m=1, \ldots, M \text {, }
$$$$
j \in \alpha_{m} t=1, \ldots, T, s \in S_{t} \mid\left\{P_{t}\right\} \text {; }
$$$$
z_{m i j s} \geq y_{m i(s-1)}+y_{m j s}-1, m=
$$$$
=1, \ldots, M, i, j \in \alpha_{m}, s=1, \ldots, N \text {; }
$$$$
\sum_{i \in \alpha_{m}} Z_{m i j s} \leq y_{m j s} m=1, \ldots, M, j \in \alpha_{m}, s=1, \ldots, N ;(24)
$$

$l_{j t}^{+}, l_{j t}^{-} \geq 0, j=1, \ldots, J, t=1, \ldots, T ; x_{m j s}, z_{m i j s} \geq$

$\geq 0 ; w_{m j s}, y_{m j s}=0 / 1, m=$

$=1, \ldots, M, i$ e $j \in \alpha_{m}, s=1, \ldots, N$

A função objetivo (16) minimiza os custos de estoque, atraso e troca. Como citado no início da Seção 3, as restrições do tipo (12) são mantidas no modelo $\mathrm{R} 1$, restrições (21). Elas garantem que, para algum item $j$, a variável de preparo $y_{m j s}$ é 1 , o que implica que sempre haverá troca entre subperíodos. Porém, a combinação das restrições (18), (19), (21) e (22) do modelo garantem que para todas as soluções factíveis, as trocas em subperíodos ociosos são do último item produzido para ele mesmo, ou seja, de um item $j$ para $j$ (característica presente na solução ótima do problema). Assim, se torna necessário anular apenas os custos/tempos de troca de $j$ para $j$ nos subperíodos ociosos (variável $Z_{\text {mijs }}$ ). 0 termo $\left(z_{\text {mijs }}-w_{\text {mjs }}\right)$ que controla as trocas entre itens iguais será nulo para os subperíodos ociosos, pois ocorrerá $y_{m j s}=1$, que implica nas restrições (18) e (19), em $w_{m j s}=1$, e nas restrições (23) em $z_{\text {mijs }}=1$, e então $z_{\text {mijs }}=w_{m j s}=1$.

A restrição (17) é a restrição de balanceamento de estoque. As restrições (18) e (19) definem os lotes mínimos e máximos de produção da linha. Se não há produção no subperíodo, o lote é nulo. Porém, pela restrição (21), para algum item $j$, a variável de preparo $y_{m j s}$ é 1, o que implica que a variável $w_{m j t}$ deve assumir valor 1 para que o termo $\left(y_{m j s}-w_{m j t}\right)$ seja nulo. A restrição (22) faz com que os subperíodos ociosos ocorram no fim do período e que a linha sempre esteja preparada para um mesmo item. Esta restrição, combinada com a restrição (21), garante que a linha é mantida preparada com o último item produzido. Nos subperíodos ociosos, a variável de setup $y_{m j s}$ assume valor 1 para um item $j$, o mesmo para o qual, pelas restrições (19), a variável $w_{m j t}$ assume valor 1. Pela ordenação das restrições (22), este item será mantido preparado em todos os subperíodos ociosos, até o fim do período. Dada a minimização dos custos de troca em (16) e as restrições (23) de troca de item, a variável de troca $Z_{\text {mijs }}$ assumirá valor 1 nos subperíodos ociosos. As restrições (24) relacionam as variáveis de troca com as variáveis de setup, e estabelecem que haja troca apenas se o item estiver preparado. Embora as restrições (24) não sejam necessárias, elas foram mantidas, pois o modelo apresentou melhores resultados em exemplares reais com estas restrições. As restrições (25) definem o domínio das variáveis.

Note que o modelo R1 possui 9 conjuntos de restrições, 5 conjuntos a menos que o modelo P2SMM. Apesar do conjunto de variáveis $w_{m j s}$ ter sido incluído, o número total de variáveis em geral é menor, uma vez que as variáveis de preparo e troca do estágio 1 foram removidas.

\subsection{Modelo monoestágio R2}

Um conjunto de restrições alternativo pode ser considerado no modelo R1, sem perda de generalidade. As restrições de troca (23) podem ser substituídas pelas restrições de balanceamento de fluxo de setup (WOLSEY, 1990):

$$
\begin{aligned}
& \sum_{i \in \alpha_{m}} Z_{m i j s}=y_{m j s}, m=1, \ldots, M, j \in \alpha_{m}, s=1, \ldots, N ; \\
& \sum_{j \in \alpha_{m}} Z_{m i j s}=y_{m i(s-1)}, m=1, \ldots, M, i \in \alpha_{m} s=2, \ldots, N ;
\end{aligned}
$$

Para $S=1$, temos $z_{m i j 1}=y_{m j 1}$; pela minimização dos custos de troca, a variável de troca escolhida é $z_{\text {mijs }}$, que possui o menor custo de troca. Esta é a troca correta que ocorre na solução ótima, pois no período inicial do horizonte de planejamento, o único preparo existente é o preparo do xarope, dado que a cada novo horizonte de planejamento, a linha está preparada. As restrições (26) garantem que apenas um item é preparado no subperíodo $s$, enquanto as restrições (27) definem o item preparado no subperíodo 
anterior. Estes conjuntos de restrições definidos para $s=2, \ldots, N$ garantem o sequenciamento dos períodos, conforme o exemplo a seguir.

Suponha a troca do item 5 para $3 \mathrm{em}$ um subperíodo $s$ qualquer. As restrições (26) ativas são aquelas em que $j=3,\left(\sum_{i \in \alpha_{m}} Z_{m i 3 s}=y_{m 3 s}=1\right)$, ou seja, haverá troca do item $i$ para o item 3 no subperíodo s. 0 valor correto de $Z_{m 53 s}$ não fica definido apenas com (26). Por exemplo, a solução $y_{m 3 s}=1$, $Z_{m 43 s}=0,5$ e $Z_{m 53 s}=0,5$ não é eliminada por aquele conjunto de restrições. Por outro lado, as restrições (27) garantem que no subperíodo anterior, em que $y_{m 5(s-1)}=1$, a única restrição ativa é aquela em que $i=5\left(\sum_{j \in \alpha_{m}} z_{m 5 j s}=y_{m 5(s-1)}=1\right)$. Assim, $z_{m i 3 s}=0$ para todo $i \neq 5$; logo, pode ocorrer apenas que $z_{m 53 s}=1$.

Denominamos o modelo alternativo definido pelas restrições (16)-(22), (25)-(27) como modelo R2. Em Ferreira (2006) estas inequações foram testadas no modelo P2SMM, mas não forneceram bons resultados. A Tabela 1 apresenta o número total de restrições dos modelos. É possível notar na tabela que os modelos R1 e R2 possuem um número menor de restrições que o modelo P2SMM. A redução do número de restrições dos modelos R1 e R2 ocorre pela ausência das restrições do estágio 1 . Os modelos R1 e R2 diferem pelas restrições de troca de bebidas nas linhas de envase. 0 modelo R2 possui $2^{*} \Sigma_{m}\left(\left|\alpha_{m}\right| . M\right)$ restrições de troca, enquanto o modelo R1 possui $\Sigma_{m}\left(\left|\alpha_{m}\right|^{2} . M\right)$ restrições do tipo (23). Assim, o modelo R2 possui em geral menos restrições que o modelo R1.
Com relação ao número de variáveis, temos que $\left|\beta_{m}\right| \leq\left|\alpha_{m}\right|$. Assim, embora as variáveis $y_{m l s}^{\prime}$ não estejam presentes nos modelos R1 e R2, o número de variáveis binárias pode ser maior nos modelos $\mathrm{R} 1$ e R2 do que no modelo P2SMM devido às variáveis $w_{\text {mis }}$ Na Tabela 2, estão apresentadas as quantidades totais de variáveis de cada modelo. 0 modelo P2SMM e os modelos R1 e R2 diferem pelas proporções, $\Sigma_{m}\left(\left|\beta_{m}\right| . M\right) .\left[\Sigma_{m}\left(\left|\beta_{m}\right|+1\right]+M . N\right.$ e $\Sigma_{m}\left(\left|\alpha_{m}\right| . M\right)$ de variáveis, respectivamente. Embora o número de variáveis binárias dos modelos R1 e R2 possa, em alguns casos, ser maior do que o número de variáveis binárias do modelo P2SMM, o número total de variáveis é, em geral, menor (Tabela 2).

\section{Experimentos computacionais}

\subsection{Exemplares de tamanho moderado}

Para comparar os desempenhos dos modelos R1 e R2 com o do modelo P2SMM, inicialmente foram utilizados 28 exemplares de tamanho moderado, baseados em dados reais de uma empresa de bebidas. Estes exemplares, designados por E1 a E28, envolvem um número menor de itens e linhas de envase do que os típicos exemplos reais da empresa e, por isso, são aqui considerados como de tamanho moderado. A motivação para estes experimentos é comparar os esforços computacionais necessários para resolver

Tabela 1. Número total de restrições dos modelos.

\begin{tabular}{cc}
\hline Modelo & Fórmula para o número total de restrições \\
\hline P2SMM & $J . T+5 . M \cdot N+\sum_{m}\left(\left|\alpha_{m}\right| \cdot N\right) \cdot\left[\sum_{m}\left|\alpha_{m}\right|+1\right]+2 \cdot \sum_{m}\left(\left|\beta_{m}\right| \cdot N\right)+\sum_{m}\left(\left|\beta_{m}\right| \cdot N\right) \cdot\left[\sum_{m}\left|\beta_{m}\right| \cdot\left(\frac{(N+T-1)}{N}\right)\right]$ \\
R1 & $J . T+M .(N+T)+\sum_{m}\left(\left|\alpha_{m}\right| \cdot N\right) \cdot\left(3-\frac{T}{N}\right)+\sum_{m}\left(\left|\alpha_{m}\right|^{2} \cdot N\right)$ \\
R2 & $J . T+M .(N+T)+\sum_{m}\left(\left|\alpha_{m}\right| \cdot N\right) \cdot\left(3-\frac{T}{N}\right)+2 \sum_{m}\left(\left|\alpha_{m}\right| \cdot N\right)$ \\
\hline
\end{tabular}

Tabela 2. Número total de variáveis dos modelos.

\begin{tabular}{cc}
\hline Modelo & Fórmula para o número total de variáveis \\
\hline P2SMM & 2.J.T $+\sum_{m}\left(\left|\alpha_{m}\right| \cdot N\right) \cdot\left[\sum_{m}\left|\alpha_{m}\right|+2\right]+\sum_{m}\left(\left|\beta_{m}\right| \cdot N\right) \cdot\left[\sum_{m}\left|\beta_{m}\right|+1\right]+M \cdot N$ \\
R1 & 2.J.T $+\sum_{m}\left(\left|\alpha_{m}\right| \cdot N\right) \cdot\left[\sum_{m}\left|\alpha_{m}\right|+2\right]+\sum_{m}\left(\left|\alpha_{m}\right| \cdot N\right)$ \\
R2 & 2.J.T $+\sum_{m}\left(\left|\alpha_{m}\right| \cdot N\right) \cdot\left[\sum_{m}\left|\alpha_{m}\right|+2\right]+\sum_{m}\left(\left|\alpha_{m}\right| \cdot N\right)$ \\
\hline
\end{tabular}


otimamente os modelos P2SMM, R1 e R2, dado que seria difícil encontrar soluções ótimas de exemplos de tamanhos mais realistas, devido aos grandes requisitos de tempos de execução e memórias computacionais.

Os exemplares de tamanho moderado possuem 2 linhas de envase para produção de 4 bebidas cada, que utilizam 2 xaropes diferentes. A programação da produção é considerada para um horizonte de 3 períodos, com 6 subperíodos em cada período. Nos exemplares E1 a E7, a maior parte dos parâmetros, como custos de estoque e atraso, tempos de troca e demandas, é baseada em dados reais. Eles diferem entre si apenas pela variação das demandas. Os exemplares E8 a E14 são baseados nos sete primeiros exemplares, mas a capacidade das linhas em cada período, $K_{m e}$ foi reduzida o suficiente para que fossem inevitáveis os atrasos na produção. Os exemplares E15 a E21 são baseados nos exemplares E8 a E14, mas nestes exemplares foram considerados custos de troca de xarope, $c_{k l}^{l}>0$, que, nos outros exemplares, estavam embutidos nos custos de troca dos itens. Os últimos sete exemplares (E22 a E28) são baseados nos exemplares E1 a E7, mas com custos de preparo de xarope como nos exemplares E15 a E21. A Tabela 3 resume as características destes exemplares testados.

Os modelos foram implementados na linguagem de modelagem AMPL 10.0 (FOURER; GAY; KERNIGHAN, 1993) e resolvidos pelo sistema de otimização CPLEX, versão 11.0 (ILOG, 2008), utilizando os parâmetros default do CPLEX. Foi utilizado um computador Pentium 4 de $2 \mathrm{~Gb}$ RAM e $3 \mathrm{GHz}$. 0 número total de variáveis, variáveis binárias e de restrições de cada modelo é apresentado na Tabela 4. É possível observar na Tabela 4 que o número total de variáveis e restrições dos modelos R1 e R2, para estes exemplares de tamanho moderado, são menores do que do modelo P2SMM, embora o número de variáveis binárias seja maior.

Tabela 3. Características dos exemplares de dimensões moderadas.

\begin{tabular}{|c|c|}
\hline $\begin{array}{l}\text { Conjunto de } \\
\text { exemplares }\end{array}$ & $\begin{array}{l}\text { Caracteristicas dos } \\
\text { exemplares }\end{array}$ \\
\hline E1a E7 & Baseadas em exemplares reais \\
\hline E8 a E14 & ldem exemplares E1 a E7, com capacidade reduzida \\
\hline E15 a E21 & $\begin{array}{l}\text { Idem exemplares E8 a E14, com custos de troca } \\
\text { de xarope }\end{array}$ \\
\hline E22 a E28 & $\begin{array}{l}\text { ldem exemplares E1 a E7, com custos de troca } \\
\text { de xarope. }\end{array}$ \\
\hline
\end{tabular}

Tabela 4. Número total de variáveis e restrições dos modelos.

\begin{tabular}{cccc}
\hline Modelo & Variáveis & Var. Binárias & Restrições \\
\hline P2SMM & 1.140 & 216 & 1.148 \\
R1 & 1.032 & 288 & 1.046 \\
R2 & 1.032 & 288 & 758 \\
\hline
\end{tabular}

Na Tabela 5, são apresentados os valores da solução ótima dos exemplares E1-E28 e os tempos de cada modelo para obtenção desta solução. Os valores em negrito são os menores tempos obtidos para se encontrar uma solução comprovadamente ótima para cada exemplar. Analisando os resultados fornecidos por cada modelo, em geral, o modelo R2 foi o melhor em 23 (dos 28) exemplares, por requerer os menores tempos do AMPL/CPLEX, enquanto o modelo P2SMM foi o melhor em apenas 6 exemplares (houve empate em um dos exemplares). Esta superioridade é mais evidente em exemplares em que os custos de troca de xarope não são contabilizados (ver resultados dos exemplares de E1 a E14), em que R2 teve o melhor desempenho em todos os 14 exemplares.

A Tabela 6 indica o número de nós avaliados; os valores em negrito são os menores valores e os valores em itálico são os maiores valores para o número total de nós avaliados pelo CPLEX em cada exemplar. Embora as dimensões dos modelos sejam próximas, nota-se que o modelo R2 necessita avaliar um número menor de nós para provar a otimalidade, estando o modelo P2SMM no extremo oposto em termos do número de nós avaliados da árvore branch and cut do CPLEX, sendo necessário, portanto, mais tempo para provar a otimalidade da solução. Quando os custos de troca de xarope são incorporados (exemplares E15 a E28), o número de nós pesquisados pelo branch and cut reduz-se de uma forma significativa para todos os modelos, e os desempenhos dos modelos P2SMM e R2 se aproximam, sobretudo nos exemplares E15 a E21 em que a capacidade de produção é reduzida.

Do ponto de vista dos valores da relaxação linear dos modelos, testes computacionais mostraram que, para os exemplares E15 a E28, a relaxação do modelo R2 é em média 2,77\% melhor que dos outros modelos (valor mínimo 2,6\% e valor máximo 3,7\%), ou seja, as restrições de troca (26) e (27) tiveram mais efeito quando há custos de troca de xarope. Para os exemplares E1 a E14, os modelos possuem os mesmos valores de relaxação linear.

\subsection{Exemplares reais da literatura}

Neste segundo conjunto de experimentos, comparamos os desempenhos dos dois modelos propostos, R1 e R2, com o modelo P2SMM nos exemplares reais $\mathrm{P} 1-\mathrm{P} 15$ analisados em Ferreira, Morabito e Rangel (2009). Para efeito de comparação com os resultados da literatura, os tempos de execução do AMPL/CPLEX foram limitados em 4 horas para cada exemplar. A Tabela 7 apresenta os números de variáveis e restrições dos modelos P2SMM, R1 e R2 nestes exemplares - note que estes números são significativamente maiores do que os da Tabela 4. 
Tabela 5. Tempos de solução (s.).

\begin{tabular}{lcrrr}
\hline Ex. & Sol. ótima & P2SMM & \multicolumn{1}{c}{ R1 } & \multicolumn{1}{c}{ R2 } \\
\hline E1 & 257,7 & $1.692,6$ & 207,2 & 128,4 \\
E2 & 264,3 & $2.565,0$ & 199,9 & 98,1 \\
E3 & 275,3 & 492,6 & 117,3 & 116,5 \\
E4 & 278,2 & $1.294,3$ & 150,3 & 137,8 \\
E5 & 260,9 & $4.309,9$ & 126,1 & 74,8 \\
E6 & 271,0 & $4.695,7$ & 109,4 & 43,2 \\
E7 & 215,3 & $3.361,4$ & 60,7 & 68,8 \\
E8 & 257,7 & 146,5 & 430,3 & 111,7 \\
E9 & 264,3 & 571,4 & 137,5 & 76,3 \\
E10 & 345,4 & 91,5 & 157,2 & 68,0 \\
E11 & 336,0 & 741,5 & 423,4 & 167,6 \\
E12 & 272,5 & 218,5 & 250,9 & 70,6 \\
E13 & 354,7 & 575,5 & 82,7 & 58,3 \\
E14 & 215,3 & 72,5 & 67,9 & 34,1 \\
E15 & $1.028,1$ & 7,0 & 61,0 & 21,0 \\
E16 & 347,7 & 16,0 & 88,0 & 24,0 \\
E17 & 720,4 & 47,0 & 88,0 & 21,0 \\
E18 & 770,9 & 6,0 & 151,0 & 26,0 \\
E19 & 658,2 & 47,0 & 49,0 & 19,0 \\
E20 & 719,7 & 12,0 & 60,0 & 14,0 \\
E21 & 910,6 & 12,0 & 53,0 & 12,0 \\
E22 & 267,7 & $1.442,0$ & 439,0 & 63,0 \\
E23 & 274,3 & 964,0 & 428,0 & 50,0 \\
E24 & 285,3 & 96,0 & 260,0 & 82,0 \\
E25 & 288,2 & 157,0 & 518,0 & 889,0 \\
E26 & 270,9 & 75,0 & 153,0 & 53,0 \\
E27 & 280,9 & 171,0 & 83,0 & 24,0 \\
E28 & 225,3 & 38,0 & 89,0 & 21,0 \\
\hline & & & & \\
\hline
\end{tabular}

As cardinalidades de $\left|\alpha_{1}\right|=23$ e $\left|\alpha_{2}\right|=10$ resultaram em 39.675 restrições de troca para a linha 1 e 7.500 para a linha 2, ou seja, 47.175 restrições de troca no modelo R1, enquanto que no modelo R2 são apenas 4.917 restrições de troca.

A Tabela 8 apresenta as soluções obtidas com os modelos nestes exemplares $\mathrm{P} 1$ a $\mathrm{P} 5$ resolvidos pelo AMPL/CPLEX no tempo limite de 4 horas. A melhor solução obtida em cada exemplar está destacada em negrito. 0 modelo R2 obteve a melhor solução nos 5 exemplares. Os gaps de otimalidade foram altos em todos os modelos (maiores que 96\%). Segundo Ferreira, Morabito e Rangel (2009), a solução da empresa para o exemplar P1 tem um custo total de 422.717, ou seja, o modelo R2 fornece uma solução com custo total menor (333.277) que a solução apresentada pela empresa, diferentemente dos modelos P2SMM e R1.

Em função das dificuldades para resolver o modelo P2SMM nestes exemplares P1-P5, Ferreira, Morabito e Rangel (2009) propuseram uma outra abordagem heurística para tratá-los, denominada Estratégia de Relaxação (ER_G21), que obteve as melhores soluções para este conjunto de exemplos. Resumidamente, em um primeiro passo, esta abordagem resolve um modelo
Tabela 6. Número de nós avaliados pelo CPLEX.

\begin{tabular}{|c|c|c|c|}
\hline Ex. & P2SMM & R1 & R2 \\
\hline E1 & 2.072 .803 & 73.953 & 60.931 \\
\hline E2 & 400.198 & 104.743 & 56.735 \\
\hline E3 & 988.210 & 32.772 & 50.964 \\
\hline E4 & 1.020 .004 & 45.778 & 66.845 \\
\hline E5 & 5.792 .046 & 419.570 & 38.240 \\
\hline E6 & 3.606 .606 & 34.034 & 23.522 \\
\hline E7 & 3.696 .541 & 20.741 & 33.271 \\
\hline E8 & 113.207 & 187.056 & 48.365 \\
\hline E9 & 452.597 & 80.498 & 37.287 \\
\hline E10 & 63.084 & 66.847 & 30.319 \\
\hline E11 & 684.209 & 209.059 & 77.292 \\
\hline E12 & 203.513 & 125.848 & 32.162 \\
\hline E13 & 597.663 & 26.822 & 26.477 \\
\hline E14 & 68.720 & 34.594 & 12.519 \\
\hline E15 & 3.337 & 3.589 & 1.919 \\
\hline E16 & 8.724 & 6.771 & 4.533 \\
\hline E17 & 2.352 & 2.950 & 2.377 \\
\hline E18 & 35.496 & 6.521 & 2.981 \\
\hline E19 & 4.986 & 3.850 & 2.519 \\
\hline E20 & 7.398 & 3.177 & 1.606 \\
\hline E21 & 7.983 & 1.638 & 1.487 \\
\hline E22 & 1.253 .963 & 23.922 & 15.099 \\
\hline E23 & 576.455 & 38.384 & 25.451 \\
\hline E24 & 51.009 & 11.081 & 32.062 \\
\hline E25 & 70.881 & 32.045 & 29.086 \\
\hline E26 & 46.794 & 9.717 & 15.688 \\
\hline E27 & 83.771 & 4.310 & 6.030 \\
\hline E28 & 33.895 & 4.525 & 5.285 \\
\hline
\end{tabular}

Tabela 7. Número total de variáveis e restrições nos exemplares reais.

\begin{tabular}{cccc}
\hline Modelo & Variáveis & Var. Binárias & Restrições \\
\hline P2SMM & 86.359 & 4.575 & 86.140 \\
R1 & 54.738 & 4.950 & 59.149 \\
R2 & 54.738 & 4.950 & 14.976 \\
\hline
\end{tabular}

Tabela 8. Custos totais das soluções dos modelos nos exemplares R1 a R5.

\begin{tabular}{rrrr}
\hline Ex. & P2SMM & \multicolumn{1}{c}{ R1 } & R2 \\
\hline P1 & 631.507 & 966.958 & 333.277 \\
P2 & 819.960 & 1.522 .085 & 378.614 \\
P3 & 817.945 & 1.009 .121 & 440.035 \\
P4 & 749.234 & 4.298 .926 & 415.388 \\
P5 & 1.038 .280 & 4.066 .081 & 517.836 \\
\hline
\end{tabular}

monoestágio com múltiplas máquinas, denominado P1SMM. Este modelo é obtido simplesmente pela eliminação de todas as variáveis e restrições do estágio 1, exceto a variável de preparo, $y_{m / s}^{l}$, e das restrições de quantidade mínima e máxima de xarope no tanque, do modelo P2SMM. Dado que a variável de espera da máquina pelo tanque, $v_{m s}^{\prime l}$, também é 
eliminada, a restrição (10) também é eliminada e a restrição de capacidade (9) é substituída por uma restrição de capacidade, em que não há o termo do tempo de espera $\sum_{s \in S_{t}} v_{m s}^{\prime \prime}$. Em um segundo passo, a solução deste modelo é fixada no modelo P2SMM, que é então resolvido. Uma heurística do tipo relax and fix, denominada heurística G21, que faz a partição por máquinas/períodos e fixa as variáveis de setup, é aplicada para resolver o modelo P1SMM.

Os desempenhos dos modelos R1 e R2 foram comparados com o da heurística ER_G21 para resolver os exemplares P1-P15. Os resultados obtidos são apresentados na Tabela 9. Convém notar que os exemplares P6-P15 utilizam os mesmos dados de custos, tempos, capacidades, etc., do exemplar P1, exceto pelos valores das demandas dos produtos. Ao contrário dos exemplares $\mathrm{P} 1$ a $\mathrm{P} 5$, que possuem no máximo um valor de demanda nulo, a partir do exemplar P6 até o exemplar P12, a matriz de demanda possui vários valores nulos, ou seja, a capacidade é mais folgada que nos exemplares P1 a P5.

Pela Tabela 9, nota-se que as soluções do modelo R2 são melhores que as do modelo R1 em todos os exemplares. Quando comparado com a estratégia heurística ER_G21, suas soluções são melhores em 5 dos 15 exemplares. Em relação ao número de nós avaliados, nos exemplares do modelo R1, o maior número de nós avaliados é 671 (exemplar P6), enquanto nos exemplares do modelo R2 são avaliados até 7.651 nós (exemplar P2). No modelo R2 avaliam-se mais nós do que no modelo $\mathrm{R} 1$ no limite de tempo de 4 horas, o que pode explicar em parte a melhor qualidade das soluções obtidas por R2. É necessário mais tempo para resolver cada nó, ou seja, o modelo é mais difícil de ser resolvido.

\subsection{Exemplares reais sem custos de troca}

Nos exemplares P1-P15, foram considerados custos de troca de bebidas nas linhas, que representam custos de oportunidade de não se produzir bebidas durante o tempo em que a linha fica parada para o preparo da próxima bebida a ser produzida. Porém, como discutido em Toso, Morabito e Clark (2009), custos de oportunidade de troca são questionáveis nos períodos em que a capacidade de produção está ociosa. Em situações em que a capacidade do período é apertada, as soluções obtidas também serão minimizadas em relação às trocas, devido à restrição de capacidade que considera os tempos de troca. Dado que os modelos R1 e R2 diferem justamente pelas restrições de troca, foram realizados testes adicionais com os exemplares P1 a P15 (agora denominados
Tabela 9. Custos totais das soluções dos modelos propostos nos exemplares P1 a P15.

\begin{tabular}{crrr}
\hline Exemplar & ER_G21 & \multicolumn{1}{c}{ R1 } & R2 \\
\hline P1 & 306.834 & 966.958 & 333.277 \\
P2 & 321.811 & 1.522 .085 & 378.614 \\
P3 & 290.841 & 1.009 .121 & 440.035 \\
P4 & 317.599 & 4.298 .926 & 415.388 \\
P5 & 379.529 & 4.066 .081 & 517.836 \\
P6 & 526.473 & 1.363 .424 & 770.111 \\
P7 & 509.464 & 1.833 .328 & 435.889 \\
P8 & 509.668 & 2.648 .909 & 425.340 \\
P9 & 412.237 & 4.692 .963 & 364.571 \\
P10 & 429.868 & 704.501 & 301.130 \\
P11 & 289.170 & 696.055 & 316.921 \\
P12 & 491.725 & 5.383 .103 & 370.827 \\
P13 & 369.540 & 667.375 & 371.917 \\
P14 & 449.511 & 1.809 .175 & 596.365 \\
P15 & 446.194 & 5.835 .839 & 556.393 \\
\hline & & &
\end{tabular}

Tabela 10. Custos obtidos para os exemplares P1a a P15a, modelo R1.

\begin{tabular}{lrrrr}
\hline Exemplares R1 & Estoque & \multicolumn{1}{c}{ Atraso } & \multicolumn{1}{c}{ Total } & Gap \% \\
\hline P1a & $11.259,8$ & $4.204,2$ & $15.464,0$ & 32,6 \\
P2a & $23.163,1$ & $7.622,6$ & $30.785,7$ & 32,2 \\
P4a & $14.941,6$ & $36.076,1$ & $51.017,7$ & 72,6 \\
P5a & $12.706,2$ & $540.236,0$ & $552.942,2$ & 98,1 \\
P6a & $9.758,6$ & $326.059,0$ & $335.817,6$ & 97,3 \\
P7a & $9.152,5$ & $467.227,0$ & $476.379,5$ & 98,2 \\
P8a & $10.513,4$ & $87.943,4$ & $98.456,8$ & 90,1 \\
P9a & $9.092,3$ & $89.222,7$ & $98.315,0$ & 91,1 \\
P10a & $9.126,3$ & 0,0 & $9.126,3$ & 10,7 \\
P11a & $8.801,0$ & $29.425,2$ & $38.226,2$ & 78,5 \\
P12a & $9.014,1$ & $18.042,3$ & $27.056,4$ & 70,1 \\
P13a & $8.568,0$ & $53.981,3$ & $62.549,3$ & 88,3 \\
P14a & $10.360,8$ & $86.587,3$ & $96.948,1$ & 91,0 \\
P15a & $9.074,3$ & $249.999,0$ & $259.073,3$ & 96,6 \\
\hline
\end{tabular}

Tabela 11. Custos obtidos para os exemplares P1a a P15a, modelo R2.

\begin{tabular}{crrrr}
\hline Exemplares R2 & Estoque & Atraso & Total & Gap \% \\
\hline P1a & $10.843,3$ & 0,0 & $10.843,3$ & 3,8 \\
P2a & $21.786,1$ & 0,0 & $21.786,1$ & 4,3 \\
P4a & $14.466,5$ & 61,8 & $14.528,3$ & 3,6 \\
P5a & $12.207,7$ & $817.236,0$ & $829.443,7$ & 98,6 \\
P6a & $9.469,8$ & $104.038,0$ & $113.507,8$ & 91,9 \\
P7a & $9.207,4$ & $95.186,1$ & $104.393,5$ & 91,6 \\
P8a & $10.468,2$ & $10.425,7$ & $20.893,9$ & 53,3 \\
P9a & $9.524,7$ & 0,0 & $9.524,7$ & 7,9 \\
P10a & $8.909,4$ & 777,7 & $9.687,1$ & 15,9 \\
P11a & $9.078,0$ & $4.996,2$ & $14.074,2$ & 41,5 \\
P12a & $9.087,8$ & $1.795,4$ & $10.883,2$ & 25,7 \\
P13a & $8.329,6$ & $14.425,1$ & $22.754,7$ & 67,8 \\
P14a & $9.506,2$ & $11.458,2$ & $20.964,4$ & 58,3 \\
P15a & $9.392,0$ & $73.247,9$ & $82.639,9$ & 89,4 \\
\hline
\end{tabular}


exemplares $\mathrm{P} 1 \mathrm{a}$ a $\mathrm{P} 15 \mathrm{a}$, respectivamente), nos quais os custos de troca foram considerados nulos.

As Tabelas 10 e 11 detalham os custos das soluções obtidas para os exemplares P1a-P15a dos modelos R1 e R2, respectivamente. A primeira coluna das tabelas apresenta o nome do exemplar, e as colunas seguintes mostram o custo de estoque, o custo de atraso, o custo total e o gap, respectivamente (os custos de troca são nulos). Os valores em negrito indicam a melhor solução obtida para cada exemplar. Como o exemplar P3 difere do exemplar P1 apenas nos custos de troca, ele não foi incluído nas Tabelas 10 e 11 porque sua solução coincide com a do exemplar P1a. Comparando os resultados das Tabelas 10 e 11, observamos que o modelo R2 ainda se mantém como o de melhor desempenho, fornecendo as melhores soluções em 13 dos 15 exemplares. Note que, na maioria deles, a diferença entre as soluções é significativa, o que reforça o bom desempenho do modelo $\mathrm{R} 2$ em relação ao modelo $\mathrm{R} 1$.

\section{Conclusões}

Este estudo apresenta duas formulações monoestágio, modelos R1 e R2, para o problema dois estágios com sincronia de dimensionamento e sequenciamento de lotes. Estes modelos foram utilizados para resolver um problema de programação da produção de bebidas multiproduto, multimáquina e tempos e custos de troca dependentes da sequência de produção. Nestas formulações, a questão da sincronia foi tratada basicamente fazendo-se um pré-processamento nos dados de tempos de troca e lotes mínimos de xarope, e adicionando-se variáveis para manter a configuração das trocas em períodos ociosos.

Os desempenhos dos modelos R1 e R2 foram comparados com o do modelo P2SMM conhecido da literatura, resolvendo-se diversos exemplares baseados em dados reais de uma fábrica de bebidas. Os resultados computacionais mostraram um bom desempenho do modelo R2 em relação aos outros dois modelos. Também mostraram que ele é competitivo com uma abordagem heuristica da literatura (ER_G21) (FERREIRA; MORABIT0; RANGEL, 2009). Uma pesquisa futura interessante seria desenvolver abordagens heurísticas baseadas nos modelos R1 e R2, como, por exemplo, heurísticas do tipo relax and fix, explorando características específicas destes modelos, para resolver este problema de programação da produção de bebidas de maneira ainda mais efetiva. Outra perspectiva interessante para pesquisa futura seria estender os modelos R1 e R2 para considerarem a possibilidade de um tanque abastecer mais de uma linha de envase por vez.

\section{Referências}

ALMADA-LOBO, B. et al. Multiple machine continuous setup lotsizing with sequence-dependent setups. Computational Optimization and Applications, v. 47, n. 3, p. 529-552, 2010. http://dx.doi.org/10.1007/ s10589-009-9235-8

ALMADA-LOBO, B.; OLIVEIRA, J. F.; CARRAVILLA, M. A. Production planning and scheduling in the glass container industry: A VNS approach. International Journal of Production Economics, vol. 114, n. 1, p. 363375, 2008. http://dx.doi.org/10.1016/j.ijpe.2007.02.052

ARAUJO, S. A.; ARENALES, M. N.; CLARK, A. R. Lot-sizing and furnace scheduling in small foundries. Computers and Operations Research, v. 35, p. 916-932, 2008. http:// dx.doi.org/10.1016/j.cor.2006.05.010

BRAHIMI, N. et al. Single item lot sizing problems. European Journal of Operational Research, v. 168, p. 1-16, 2006. http://dx.doi.org/10.1016/j.ejor.2004.01.054

CASTRO, J. G.; PIZZOLATO, N. D. A programação de lotes econômicos de produção (ELSP) com tempos e custos de setup dependentes da sequência: um estudo de caso. Revista Gestão Industrial, v. 1, 70-80, 2005.

CHENG, T. C. E.; DING, Q.; LIN; B. M. T. A concise survey of scheduling with time-dependent processing time. European Journal of Operational Research, v. 152, p. 1-13, 2004. http://dx.doi.org/10.1016/S03772217(02)00909-8

CLARK, A. R. Hybrid heuristics for planning lot setups and sizes. Computers \& Industrial Engineering, v. 45, p. 545-562, 2003. http://dx.doi.org/10.1016/S03608352(03)00073-1

CLARK, A. R.; CLARK, S. J. Rolling-horizon lot-sizing when setup times are sequence-dependent. International Journal of Production Research, v. 38, p. 2287-2307, 2000. http://dx.doi.org/10.1080/00207540050028106

CLARK, A. R.; MORABITO, R.; TOSO, E. Production setupsequencing and lot-sizing at an animal nutrition plant through ATSP subtour elimination and patching. Journal of Scheduling, v. 13, p. 111-121, 2010. http://dx.doi. org/10.1007/s10951-009-0135-7

DREXL, A.; KIMMS, A. Lot sizing and scheduling - survey and extensions. European Journal of Operational Research, v. 99, p. 221-235, 1997. http://dx.doi.org/10.1016/ S0377-2217(97)00030-1

FERREIRA, D. Abordagens para o problema integrado de dimensionamento e sequenciamento de lotes da produção de bebidas. 2006. Tese (Doutorado)Universidade Federal de São Carlos.

FERREIRA, D. et. al. Heuristics and metaheuristics for lot sizing and scheduling in the soft drinks industry: a comparison study, Studies in Computational Intelligence. SpringerVerlag Berlin Heidelberg, v. 128, p. 169-210, 2008.

FERREIRA, D.; MORABITO, R.; RANGEL, S. Um modelo de otimização inteira mista e heurísticas relax and fix para a programação da produção de fábricas de refrigerantes de pequeno porte. Produção, v. 18, n. 1, p. 76-88, 2008. http://dx.doi.org/10.1590/S0103-65132008000100006

FERREIRA, D.; MORABITO, R.; RANGEL, S. Solution approaches for the soft drink integrated production lot sizing and scheduling problem. European Journal of Operational Research, v. 196, n. 2, p. 697-706, 2009. http://dx.doi.org/10.1016/j.ejor.2008.03.035 
FERREIRA, D.; MORABITO, R.; RANGEL, S. Relax and fix heuristics to solve one-stage one-machine lot-scheduling models for small-scale soft drink plants. Computers \& Operations Research, v. 37, p. 684-691, 2010. http:// dx.doi.org/10.1016/j.cor.2009.06.007

FLEISCHMANN, B.; MEYR, $\mathrm{H}$. The general lotsizing and scheduling problem. OR Spektrum, v. 19, p. 11-21, 1997. http://dx.doi.org/10.1007/BF01539800

FOURER, R.; GAY, M. D.; KERNIGHAN, B. W. AMPL - a modeling language for mathematical programming. Danvers, Massachusetts: The Scientific Press, 1993.

FRANÇA, P. M; ARMENTANO, V. A.; TOLEDO, F. M. B. A network flow model for capacitated lot sizing problem. Omega-International Journal Of Management Science, v. 27, n. 2, p. 275-284, 1999. http://dx.doi.org/10.1016/ S0305-0483(98)00045-0

ILOG. Using the CPLEX Callable Library. Copyright, ILOG, 2008.

KARIMI, B.; GHOMI, S. M. T. F.; WILSON, J. M. The capacitated lot sizing problem: a review of models and algorithms. Omega International Journal of Management Science, v. 31, n. 5, p. 365-378, 2003. http://dx.doi.org/10.1016/ S0305-0483(03)00059-8

LUCHE, J. R.; MORABITO, R.; PUREZA, V. Combining process selection and lot sizing models for production scheduling of electrofused grains. Asia-Pacific Journal of Operational Research, v. 26, n. 3, p. 421-443, 2008. http://dx.doi.org/10.1142/S0217595909002286

MANNE, A. S. On the job-shop scheduling problem. Operations Research, v. 8, p. 219-223, 1960. http:// dx.doi.org/10.1287/opre.8.2.219

PINED0, M. Scheduling: theory, algorithms and systems. Prentice Hall, 1995.

RANGEL, S.; FERREIRA, D. Um modelo de dimensionamento de lotes para uma fábrica de refrigerantes. TEMA Tendências em Matemática Aplicada e Computacional, v. 4, n. 2, p. 237-246, 2003.
SANTOS-MEZA, E.; SANTOS, M. 0.; ARENALES, M. N. A lot-sizing problem in an automated foundry. European Journal of Operational Research, v. 139, p. 490-500, 2002. http://dx.doi.org/10.1016/S03772217(01)00196-5

TOLEDO, F. M. B.; ARMENTANO, V. A. A lagrangian-based heuristic for the capacitated lot-sizing problem in parallel machines. European Journal of Operational Research, v. 175, p. 1070-1083, 2006. http://dx.doi.org/10.1016/j. ejor.2005.06.029

TOLEDO, C. F. M. et al. Multi-population genetic algorithm to solve the synchronized and integrated two-level lot sizing and scheduling problem. International Journal of Production Research, v. 47, n. 11, p. 3097-3119, 2008. http://dx.doi.org/10.1080/00207540701675833

TOLEDO, C. F. M. et al. Um modelo de otimização para o problema integrado de dimensionamento de lotes e programação da produção em fábrica de refrigerantes. Pesquisa Operacional, v. 27, p. 155-186, 2007.

TOSO, E. V.; MORABITO, R.; CLARK, A. R. Lot sizing and sequencing optimization at an animal-feed plant. Computers \& Industrial Engineering, v. 57, n. 3, p. 813821, 2009. http://dx.doi.org/10.1016/j.cie.2009.02.011

WOLSEY, L. A. Valid inequalities for 0-1 knapsack and MIPs with generalized upper bound constraints. Discrete Applied Mathematics, v. 29, p. 251-261, 1990. http:// dx.doi.org/10.1016/0166-218X(90)90148-6

\section{Agradecimentos}

Os autores agradecem aos revisores os úteis comentários e sugestões; também à FAPESP, CNPq e ao 7PQ da Comissão Europeia, call Marie Curie, projeto $\mathrm{n}^{\circ} 246881$ o apoio financeiro.

\title{
Mono-stage formulations for the soft drink production process with two synchronized stages
}

\begin{abstract}
In this work we present single-stage formulations for the integrated soft drink lot-sizing and scheduling problem with two-stage synchronization. It is a multi-product, multi-machine problem, with sequence-dependent setup times and costs. Without loss of generality, these single-stage reformulations address the problem correctly and, in general, reduce the size of the synchronized two-stage model of Ferreira, Morabito e Rangel (2009), regarding the number of variables and constraints. The preliminary computational experiments on real-world instances from a soft-drink company show the competitiveness of the single-stage models against other formulations and solution approaches reported in the literature.
\end{abstract}

\section{Keywords}

Integrated lot-sizing and sequencing. Soft drink production scheduling. Mixed integer programming. Mathematical programming. 